\title{
A CLASSIFICATION OF THE TORSION TENSORS ON ALMOST CONTACT MANIFOLDS WITH B-METRIC
}

\author{
MANCHO MANEV AND MIROSLAVA IVANOVA
}

\begin{abstract}
The space of the torsion (0,3)-tensors of the linear connections on almost contact manifolds with B-metric is decomposed in 15 orthogonal and invariant subspaces with respect to the action of the structure group. Three known connections, preserving the structure, are characterized regarding this classification.
\end{abstract}

\section{INTRODUCTION}

The investigations of linear connections on almost contact manifolds with B-metric take a central place in the study of the differential geometry of these manifolds. The linear connections preserving the metric are completely characterized by their torsion tensors. In accordance with our goals, it is important to describe linear connections regarding the properties of their torsion tensors with respect to the structures on the manifold.

Such a classification of the space of the torsion tensors is made in [10] in the case of almost complex manifolds with Norden metric. These manifolds are the even-dimensional analogue of the odd-dimensional almost contact manifolds.

The idea of decomposition of the space of the basic (0,3)-tensors, generated by the covariant derivative of the fundamental tensor of type $(1,1)$, is used by different authors in order to obtain classifications of manifolds with additional tensor structures. For example, let us mention the classification of almost Hermitian manifolds given in 14, of almost complex manifolds with Norden metric - in [7], of almost contact metric manifolds - in [1], of almost contact manifolds with B-metric - in [11], of Riemannian almost product manifolds - in [32, of Riemannian manifolds with traceless almost product structure - in [33], of almost paracontact metric manifolds - in [31], of almost paracontact Riemannian manifolds of type $(n, n)-$ in $[28]$.

The linear connections preserving the structure (also known as natural connections) are particularly interesting in differential geometry. On an almost Hermitian manifold there exists a unique natural connection $\nabla^{C}$ with a torsion $T$ which has the property $T(J \cdot, J \cdot)=-T(\cdot, \cdot)$ with respect to the almost complex structure $J$. This connection is known as the canonical Hermitian connection or the Chern connection [4, 38, 39]. An example of the natural Hermitian connection is the first canonical connection of Lichnerowicz $\nabla^{L}$ [20, 21]. According to [13, there exists a one-parameter family of canonical Hermitian connections $\nabla^{t}=t \nabla^{C}+(1-t) \nabla^{L}$. The connection $\nabla^{t}$ obtained for $t=-1$ is called the Bismut connection or the KT-connection, which is characterized

2000 Mathematics Subject Classification. Primary 53C05; Secondary 53C15, 53C50.

Key words and phrases. torsion, almost contact manifold, B-metric, natural connection. 
with a totally skew-symmetric torsion [2]. The latter connection has applications in heterotic string theory and in 2-dimensional supersymmetric $\sigma$-models as well as in type II string theory when the torsion 3 -form is closed [12, 34, 19, 18]. In [5] and [6] all almost Hermitian and almost contact metric structures admitting a connection with totally skew-symmetric torsion tensor are described. Natural connections of canonical type are considered on the Riemannian almost product manifolds in [15, 16, 17] and on the almost complex manifolds with Norden metric in [10, 8, 30. The Tanaka-Webster connection on a contact metric manifold is introduced ([36, 35, 37]) in the context of CR-geometry. A natural connection with minimal torsion on the quaternionic contact structures, introduced in [3], is known as the Biquard connection.

The goal of the present work is to describe the torsion space with respect to the almost contact B-metric structure, which could be used to study some natural connections on these manifolds.

This paper is organized as follows. In Sec. 1, we present some necessary facts about the considered manifolds. Sec. 2 is devoted to the decomposition of the space of torsion tensors on almost contact manifolds with B-metric. In Sec. 3, we find the position of three known natural connections in the obtained classification.

\section{Convention 1.}

(a) We shall use $X, Y, Z$ to denote elements of of the algebra $\mathfrak{X}(M)$ on the smooth vector fields on $M$. Moreover, $x, y, z$ will stand for arbitrary vectors in the tangent space $T_{p} M$ of $M$ at an arbitrary point $p$ in $M$;

(b) The notation $\underset{x, y, z}{\mathfrak{S}}$ means the cyclic sum by the three arguments $x, y, z$. For example, $\underset{x, y, z}{\mathfrak{S}} F(x, y, z)=F(x, y, z)+F(y, z, x)+F(z, x, y)$

(c) For the sake of brevity, we shall use the notation $\{A(x, y)\}_{[x \leftrightarrow y]}$ for the difference $A(x, y)-A(y, x)$ and $\{A(x, y)\}_{(x \leftrightarrow y)}$ for the sum $A(x, y)+A(y, x)$, where $A$ is an arbitrary tensor. Similarly, we use $\{A(x, y, z)\}_{[x \leftrightarrow y]}=A(x, y, z)-A(y, x, z)$ and $\{A(x, y, z)\}_{(x \leftrightarrow y)}=A(x, y, z)+A(y, x, z)$ for any tensor $A(x, y, z)$

(d) We shall use double subscripts separated by the symbol /. The former and latter subscripts regarding this symbol correspond to the upper and down signs plus or minus in the same equality, respectively. For example, the notation $\mathcal{F}_{8 / 9}: F(x, y, z)=F(x, y, \xi) \eta(z)+F(x, z, \xi) \eta(y), F(x, y, \xi)= \pm F(y, x, \xi)=$ $F(\varphi x, \varphi y, \xi)$ means $\mathcal{F}_{8}: F(x, y, z)=F(x, y, \xi) \eta(z)+F(x, z, \xi) \eta(y), F(x, y, \xi)=$ $F(y, x, \xi)=F(\varphi x, \varphi y, \xi)$ and $\mathcal{F}_{9}: F(x, y, z)=F(x, y, \xi) \eta(z)+F(x, z, \xi) \eta(y)$, $F(x, y, \xi)=-F(y, x, \xi)=F(\varphi x, \varphi y, \xi)$. Similarly, $\mathcal{W}_{1,1 / 1,2}=\left\{T \in \mathcal{W}_{1}^{-} \mid L_{1,1}(T)\right.$ $=\mp T\}$ means $\mathcal{W}_{1,1}=\left\{T \in \mathcal{W}_{1}^{-} \mid L_{1,1}(T)=-T\right\}$ and $\mathcal{W}_{1,2}=\left\{T \in \mathcal{W}_{1}^{-} \mid\right.$ $\left.L_{1,1}(T)=T\right\}$.

\section{Almost Contact Manifolds with B-Metric}

Let $(M, \varphi, \xi, \eta, g)$ be an almost contact manifold with B-metric or an almost contact Bmetric manifold, i.e. $M$ is a $(2 n+1)$-dimensional differentiable manifold with an almost contact structure $(\varphi, \xi, \eta)$ consisting of an endomorphism $\varphi$ of the tangent bundle, a vector field $\xi$, its dual 1 -form $\eta$ as well as $M$ is equipped with a pseudo-Riemannian 
metric $g$ of signature $(n, n+1)$, such that the following algebraic relations are satisfied: $\varphi \xi=0, \varphi^{2}=-\operatorname{Id}+\eta \otimes \xi, \eta \circ \varphi=0, \eta(\xi)=1, g(\varphi X, \varphi Y)=-g(X, Y)+\eta(X) \eta(Y)[11$.

The associated metric $\widetilde{g}$ of $g$ on $M$ is defined by $\widetilde{g}(X, Y)=g(X, \varphi Y)+\eta(X) \eta(Y)$. The manifold $(M, \varphi, \xi, \eta, \widetilde{g})$ is also an almost contact B-metric manifold. Both metrics $g$ and $\widetilde{g}$ are necessarily of signature $(n, n+1)$. The Levi-Civita connection of $g$ and $\widetilde{g}$ will be denoted by $\nabla$ and $\widetilde{\nabla}$, respectively.

Let us remark that the $2 n$-dimensional contact distribution $H=\operatorname{ker}(\eta)$, generated by the contact 1-form $\eta$, can be considered as the horizontal distribution of the sub-Riemannian manifold $M$. Then $H$ is endowed with an almost complex structure determined as $\left.\varphi\right|_{H}$ - the restriction of $\varphi$ on $H$, as well as a Norden metric $\left.g\right|_{H}$, i.e. $\left.g\right|_{H}\left(\left.\left.\varphi\right|_{H^{*}, \varphi} \varphi\right|_{H^{*}}\right)=$ $-\left.g\right|_{H}(\cdot, \cdot)$. Moreover, $H$ can be considered as a $n$-dimensional complex Riemannian manifold with a complex Riemannian metric $g^{\mathbb{C}}=\left.g\right|_{H}+\left.i \widetilde{g}\right|_{H}[9$.

The structure group of $(M, \varphi, \xi, \eta, g)$ is $\mathcal{G} \times \mathcal{I}$, where $\mathcal{I}$ is the identity on $\operatorname{span}(\xi)$ and $\mathcal{G}=\mathcal{G} \mathcal{L}(n ; \mathbb{C}) \cap \mathcal{O}(n, n)$, i.e. it consists of the real square matrices of order $2 n+1$ of the following type

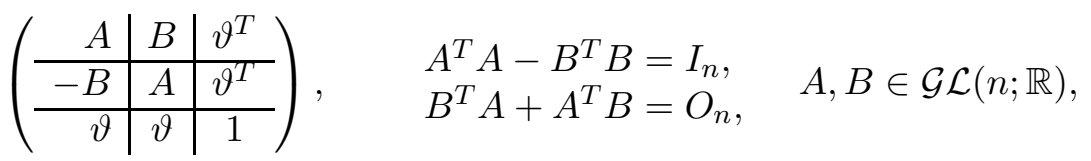

where $\vartheta$ and its transpose $\vartheta^{T}$ are the zero row $n$-vector and the zero column $n$-vector; $I_{n}$ and $O_{n}$ are the unit matrix and the zero matrix of size $n$, respectively.

A classification of almost contact manifolds with B-metric is given in [11. This classification, consisting of eleven basic classes $\mathcal{F}_{1}, \mathcal{F}_{2}, \ldots, \mathcal{F}_{11}$, is made with respect to the tensor $F$ of type $(0,3)$ defined by $F(x, y, z)=g\left(\left(\nabla_{x} \varphi\right) y, z\right)$ and having the following properties $F(x, y, z)=F(x, z, y)=F(x, \varphi y, \varphi z)+\eta(y) F(x, \xi, z)+\eta(z) F(x, y, \xi)$.

If $\left\{e_{i} ; \xi\right\} \quad(i=1,2, \ldots, 2 n)$ is a basis of $T_{p} M$ and $\left(g^{i j}\right)$ is the inverse matrix of $\left(g_{i j}\right)$, then the following 1-forms are associated with $F: \theta(z)=g^{i j} F\left(e_{i}, e_{j}, z\right), \theta^{*}(z)=$ $g^{i j} F\left(e_{i}, \varphi e_{j}, z\right), \omega(z)=F(\xi, \xi, z)$.

Further we use the following characteristic conditions of the basic classes:

$$
\begin{aligned}
\mathcal{F}_{1}: & F(x, y, z)=\frac{1}{2 n}\left\{g(x, \varphi y) \theta(\varphi z)+g(\varphi x, \varphi y) \theta\left(\varphi^{2} z\right)\right\}_{(y \leftrightarrow z)} ; \\
\mathcal{F}_{2}: & F(\xi, y, z)=F(x, \xi, z)=0, \quad \underset{x, y, z}{\mathfrak{S}} F(x, y, \varphi z)=0, \quad \theta=0 ; \\
\mathcal{F}_{3}: & F(\xi, y, z)=F(x, \xi, z)=0, \quad \underset{x, y, z}{\mathfrak{S}} F(x, y, z)=0 ; \\
\mathcal{F}_{4}: & F(x, y, z)=-\frac{1}{2 n} \theta(\xi)\{g(\varphi x, \varphi y) \eta(z)+g(\varphi x, \varphi z) \eta(y)\} \\
\mathcal{F}_{5}: & F(x, y, z)=-\frac{1}{2 n} \theta^{*}(\xi)\{g(x, \varphi y) \eta(z)+g(x, \varphi z) \eta(y)\} \\
\mathcal{F}_{6 / 7}: & F(x, y, z)=F(x, y, \xi) \eta(z)+F(x, z, \xi) \eta(y), \\
& F(x, y, \xi)= \pm F(y, x, \xi)=-F(\varphi x, \varphi y, \xi), \quad \theta=\theta^{*}=0 ; \\
\mathcal{F}_{8 / 9}: & F(x, y, z)=F(x, y, \xi) \eta(z)+F(x, z, \xi) \eta(y), \\
& F(x, y, \xi)= \pm F(y, x, \xi)=F(\varphi x, \varphi y, \xi) \\
\mathcal{F}_{10}: & F(x, y, z)=F(\xi, \varphi y, \varphi z) \eta(x) ; \\
\mathcal{F}_{11}: & F(x, y, z)=\eta(x)\{\eta(y) \omega(z)+\eta(z) \omega(y)\} .
\end{aligned}
$$


The intersection of the basic classes is the special class $\mathcal{F}_{0}$ determined by the condition $F(x, y, z)=0$. Hence $\mathcal{F}_{0}$ is the class of almost contact B-metric manifolds with $\nabla$-parallel structures, i.e. $\nabla \varphi=\nabla \xi=\nabla \eta=\nabla g=\nabla \tilde{g}=0$.

\subsection{Associated tensor of the Nijenhuis tensor.}

The Nijenhuis tensor of the contact structure is defined by $N=[\varphi, \varphi]+\mathrm{d} \eta \otimes \xi$, where $[\varphi, \varphi](x, y)=[\varphi x, \varphi y]+\varphi^{2}[x, y]-\varphi[\varphi x, y]-\varphi[x, \varphi y]$ is the Nijenhuis torsion of $\varphi$ and $\mathrm{d} \eta$ is the exterior derivative of the 1-form $\eta$.

By analogy with the skew-symmetric Lie bracket $[x, y]=\nabla_{x} y-\nabla_{y} x$, let us consider the symmetric bracket $\{x, y\}=\nabla_{x} y+\nabla_{y} x$. Then we introduce the symmetric tensor $\{\varphi, \varphi\}(x, y)=\{\varphi x, \varphi y\}+\varphi^{2}\{x, y\}-\varphi\{\varphi x, y\}-\varphi\{x, \varphi y\}$. Additionally, we use the Lie derivative of the metric $g$ along $\xi$, i.e. $\left(\mathcal{L}_{\xi} g\right)(x, y)=\left(\nabla_{x} \eta\right) y+\left(\nabla_{y} \eta\right) x$, as an alternative of $\mathrm{d} \eta(x, y)=\left(\nabla_{x} \eta\right) y-\left(\nabla_{y} \eta\right) x$. Then, we define an associated tensor $\widehat{N}$ with $N$ by:

$$
\widehat{N}=\{\varphi, \varphi\}+\left(\mathcal{L}_{\xi} g\right) \otimes \xi .
$$

It is well known that the Nijenhuis tensor $N$ is determined by covariant derivatives of $\varphi$ and $\eta$ with respect to $\nabla$ as follows:

$$
N(x, y)=\left\{\left(\nabla_{\varphi x} \varphi\right) y-\varphi\left(\nabla_{x} \varphi\right) y+\left(\nabla_{x} \eta\right) y \cdot \xi\right\}_{[x \leftrightarrow y]} .
$$

Proposition 1.1. The tensor $\widehat{N}$ has the following form in terms of $\nabla \varphi$ and $\nabla \eta$ :

$$
\widehat{N}(x, y)=\left\{\left(\nabla_{\varphi x} \varphi\right) y-\varphi\left(\nabla_{x} \varphi\right) y+\left(\nabla_{x} \eta\right) y \cdot \xi\right\}_{(x \leftrightarrow y)} .
$$

Proof. We obtain immediately

$$
\begin{aligned}
\widehat{N}(x, y)= & \{\varphi, \varphi\}(x, y)+\left(\mathcal{L}_{\xi} g\right)(x, y) \cdot \xi=\{\varphi x, \varphi y\}+\varphi^{2}\{x, y\}-\varphi\{\varphi x, y\}-\varphi\{x, \varphi y\} \\
& +\left(\nabla_{x} \eta\right) y \cdot \xi+\left(\nabla_{y} \eta\right) x \cdot \xi=\nabla_{\varphi x} \varphi y+\nabla_{\varphi y} \varphi x+\varphi^{2} \nabla_{x} y+\varphi^{2} \nabla_{y} x-\varphi \nabla_{\varphi x} y \\
& -\varphi \nabla_{y} \varphi x-\varphi \nabla_{x} \varphi y-\varphi \nabla_{\varphi y} x+\left(\nabla_{x} \eta\right) y \cdot \xi+\left(\nabla_{y} \eta\right) x \cdot \xi \\
= & \left(\nabla_{\varphi x} \varphi\right) y+\left(\nabla_{\varphi y} \varphi\right) x-\varphi\left(\nabla_{x} \varphi\right) y-\varphi\left(\nabla_{y} \varphi\right) x+\left(\nabla_{x} \eta\right) y \cdot \xi+\left(\nabla_{y} \eta\right) x \cdot \xi,
\end{aligned}
$$

which completes the proof.

It is known that the class of the normal almost contact B-metric manifolds, i.e. $N=0$, is $\mathcal{F}_{1} \oplus \mathcal{F}_{2} \oplus \mathcal{F}_{4} \oplus \mathcal{F}_{5} \oplus \mathcal{F}_{6}$.

Proposition 1.2. The class of the almost contact B-metric manifolds with $\widehat{N}=0$ is $\mathcal{F}_{3} \oplus \mathcal{F}_{7}$.

Proof. By virtue of (44) and the form of $F(x, y, z)=g\left(\left(\nabla_{x} \varphi\right) y, z\right)$ in (1), we establish that $\widehat{N}$ has the following form on $M=(M, \varphi, \xi, \eta, g)$ belonging to $\mathcal{F}_{i}(i=1,2, \ldots, 11)$, respectively:

$$
\begin{aligned}
& \widehat{N}(x, y)=\frac{2}{n}\{g(\varphi x, \varphi y) \varphi \Theta+g(x, \varphi y) \Theta\}, \quad M \in \mathcal{F}_{1} ; \\
& \widehat{N}(x, y)=2\left\{\left(\nabla_{\varphi x} \varphi\right) y-\varphi\left(\nabla_{x} \varphi\right) y\right\}, \quad M \in \mathcal{F}_{2} ; \\
& \widehat{N}(x, y)=0, \quad M \in \mathcal{F}_{3} \oplus \mathcal{F}_{7} ; \\
& \widehat{N}(x, y)=\frac{2}{n} \theta(\xi) g(x, \varphi y) \cdot \xi, \quad M \in \mathcal{F}_{4} \text {; }
\end{aligned}
$$




$$
\begin{array}{llrl}
\widehat{N}(x, y) & =-\frac{2}{n} \theta^{*}(\xi) g(\varphi x, \varphi y) \cdot \xi, & & M \in \mathcal{F}_{5} ; \\
\widehat{N}(x, y) & =4\left(\nabla_{x} \eta\right) y \cdot \xi, & & M \in \mathcal{F}_{6} ; \\
\widehat{N}(x, y) & =-2\left\{\eta(x) \nabla_{y} \xi+\eta(y) \nabla_{x} \xi\right\}, & & M \in \mathcal{F}_{8} \oplus \mathcal{F}_{9} ; \\
\widehat{N}(x, y) & =-\left\{\eta(x) \varphi\left(\nabla_{\xi} \varphi\right) y+\eta(y) \varphi\left(\nabla_{\xi} \varphi\right) x\right\}, & & M \in \mathcal{F}_{10} ; \\
\widehat{N}(x, y) & =-2 \eta(x) \eta(y) \varphi \Omega+\{\eta(x) \omega(\varphi y)+\eta(y) \omega(\varphi x)\} \cdot \xi, & & M \in \mathcal{F}_{11},
\end{array}
$$

where $\theta(z)=g(\Theta, z)$ and $\omega(z)=g(\Omega, z)$. Then the truthfulness of the statement follows.

\section{A Decomposition of the Space of Torsion Tensors}

The object of our considerations are the linear connections with torsion. Thus, we have to study the properties of the torsion tensors with respect to the contact structure and the B-metric.

If $T$ is the torsion tensor of $D$, i.e. $T(x, y)=D_{x} y-D_{y} x-[x, y]$, then the corresponding tensor of type $(0,3)$ is determined by $T(x, y, z)=g(T(x, y), z)$.

Let us consider $T_{p} M$ at arbitrary $p \in M$ as a $(2 n+1)$-dimensional vector space with almost contact B-metric structure $(V, \varphi, \xi, \eta, g)$. Moreover, let $\mathcal{T}$ be the vector space of all tensors $T$ of type $(0,3)$ over $V$ having skew-symmetry by the first two arguments, i.e.

$$
\mathcal{T}=\{T(x, y, z) \in \mathbb{R}, x, y, z \in V \mid T(x, y, z)=-T(y, x, z)\} .
$$

The metric $g$ induces an inner product $\langle\cdot, \cdot\rangle$ on $\mathcal{T}$ defined by $\left\langle T_{1}, T_{2}\right\rangle=g^{i q} g^{j r} g^{k s}$ $T_{1}\left(e_{i}, e_{j}, e_{k}\right) T_{2}\left(e_{q}, e_{r}, e_{s}\right)$ for any $T_{1,2} \in \mathcal{T}$ and a basis $\left\{e_{i}\right\}(i=1,2, \ldots, 2 n+1)$ of $V$.

The standard representation of the structure group $\mathcal{G} \times \mathcal{I}$ in $V$ induces a natural representation $\lambda$ of $\mathcal{G} \times \mathcal{I}$ in $\mathcal{T}$ as follows $((\lambda a) T)(x, y, z)=T\left(a^{-1} x, a^{-1} y, a^{-1} z\right)$ for any $a \in \mathcal{G} \times \mathcal{I}$ and $T \in \mathcal{T}$, so that $\left\langle(\lambda a) T_{1},(\lambda a) T_{2}\right\rangle=\left\langle T_{1}, T_{2}\right\rangle, T_{1}, T_{2} \in \mathcal{T}$.

The decomposition $x=-\varphi^{2} x+\eta(x) \xi$ generates the projectors $h$ and $v$ on $V$ determined by $h(x)=-\varphi^{2} x$ and $v(x)=\eta(x) \xi$ and having the properties $h \circ h=h, v \circ v=v, h \circ v=$ $v \circ h=0$. Therefore, we have the orthogonal decomposition $V=h(V) \oplus v(V)$.

Bearing in mind these projectors on $V$, we construct a partial decomposition of $\mathcal{T}$ as follows.

At first, we define the operator $p_{1}: \mathcal{T} \rightarrow \mathcal{T}$ by

$$
p_{1}(T)(x, y, z)=-T\left(\varphi^{2} x, \varphi^{2} y, \varphi^{2} z\right), \quad T \in \mathcal{T} .
$$

It is easy to check the following

Lemma 2.1. The operator $p_{1}$ has the following properties:

(i) $p_{1} \circ p_{1}=p_{1}$

(ii) $\left\langle p_{1}\left(T_{1}\right), T_{2}\right\rangle=\left\langle T_{1}, p_{1}\left(T_{2}\right)\right\rangle, \quad T_{1}, T_{2} \in \mathcal{T}$;

(iii) $p_{1} \circ(\lambda a)=(\lambda a) \circ p_{1}$.

According to Lemma 2.1 we have the following orthogonal decomposition of $\mathcal{T}$ by the image and the kernel of $p_{1}$ :

$$
\mathcal{W}_{1}=\operatorname{im}\left(p_{1}\right)=\left\{T \in \mathcal{T} \mid p_{1}(T)=T\right\}, \quad \mathcal{W}_{1}^{\perp}=\operatorname{ker}\left(p_{1}\right)=\left\{T \in \mathcal{T} \mid p_{1}(T)=0\right\} .
$$

Further, we consider the operator $p_{2}: \mathcal{W}_{1}^{\perp} \rightarrow \mathcal{W}_{1}^{\perp}$, defined by

$$
p_{2}(T)(x, y, z)=\eta(z) T\left(\varphi^{2} x, \varphi^{2} y, \xi\right), \quad T \in \mathcal{W}_{1}^{\perp} .
$$


We obtain immediately the truthfulness of the following

Lemma 2.2. The operator $p_{2}$ has the following properties:

(i) $p_{2} \circ p_{2}=p_{2}$;

(iii) $p_{2} \circ(\lambda a)=(\lambda a) \circ p_{2}$.

(ii) $\left\langle p_{2}\left(T_{1}\right), T_{2}\right\rangle=\left\langle T_{1}, p_{2}\left(T_{2}\right)\right\rangle, \quad T_{1}, T_{2} \in \mathcal{W}_{1}^{\perp} ;$

Then, bearing in mind Lemma 2.2, we obtain

$\mathcal{W}_{2}=\operatorname{im}\left(p_{2}\right)=\left\{T \in \mathcal{W}_{1}^{\perp} \mid p_{2}(T)=T\right\}, \quad \mathcal{W}_{2}^{\perp}=\operatorname{ker}\left(p_{2}\right)=\left\{T \in \mathcal{W}_{1}^{\perp} \mid p_{2}(T)=0\right\}$.

Finally, we consider the operator $p_{3}: \mathcal{W}_{2}^{\perp} \rightarrow \mathcal{W}_{2}^{\perp}$ defined by

$$
p_{3}(T)(x, y, z)=\eta(x) T\left(\xi, \varphi^{2} y, \varphi^{2} z\right)+\eta(y) T\left(\varphi^{2} x, \xi, \varphi^{2} z\right), \quad T \in \mathcal{W}_{2}^{\perp}
$$

and we get the following

Lemma 2.3. The operator $p_{3}$ has the following properties:

(i) $p_{3} \circ p_{3}=p_{3}$;

(ii) $\left\langle p_{3}\left(T_{1}\right), T_{2}\right\rangle=\left\langle T_{1}, p_{3}\left(T_{2}\right)\right\rangle, \quad T_{1}, T_{2} \in \mathcal{W}_{2}^{\perp}$

(iii) $p_{3} \circ(\lambda a)=(\lambda a) \circ p_{3}$.

By virtue of Lemma 2.3, we have

$\mathcal{W}_{3}=\operatorname{im}\left(p_{3}\right)=\left\{T \in \mathcal{W}_{2}^{\perp} \mid p_{3}(T)=T\right\}, \quad \mathcal{W}_{4}=\operatorname{ker}\left(p_{3}\right)=\left\{T \in \mathcal{W}_{2}^{\perp} \mid p_{3}(T)=0\right\}$.

From Lemma 2.1, Lemma 2.2 and Lemma 2.3 we have immediately

Theorem 2.4. The decomposition $\mathcal{T}=\mathcal{W}_{1} \oplus \mathcal{W}_{2} \oplus \mathcal{W}_{3} \oplus \mathcal{W}_{4}$ is orthogonal and invariant under the action of $\mathcal{G} \times \mathcal{I}$. The subspaces $\mathcal{W}_{i}(i=1,2,3,4)$ are determined by

$$
\begin{aligned}
& \quad \mathcal{W}_{1}: \quad T(x, y, z)=-T\left(\varphi^{2} x, \varphi^{2} y, \varphi^{2} z\right), \quad \mathcal{W}_{2}: \quad T(x, y, z)=\eta(z) T\left(\varphi^{2} x, \varphi^{2} y, \xi\right), \\
& (5) \quad \mathcal{W}_{3}: T(x, y, z)=\eta(x) T\left(\xi, \varphi^{2} y, \varphi^{2} z\right)+\eta(y) T\left(\varphi^{2} x, \xi, \varphi^{2} z\right), \\
& \mathcal{W}_{4}: T(x, y, z)=-\eta(z)\left\{\eta(y) T\left(\varphi^{2} x, \xi, \xi\right)+\eta(x) T\left(\xi, \varphi^{2} y, \xi\right)\right\} \\
& \text { for arbitrary vectors } x, y, z \in V .
\end{aligned}
$$

Corollary 2.5. The subspaces $\mathcal{W}_{i}(i=1,2,3,4)$ are characterized as follows:

$$
\begin{aligned}
& \mathcal{W}_{1}=\{T \in \mathcal{T} \mid T(v(x), y, z)=T(x, y, v(z))=0\}, \\
& \mathcal{W}_{2}=\{T \in \mathcal{T} \mid T(v(x), y, z)=T(x, y, h(z))=0\}, \\
& \mathcal{W}_{3}=\{T \in \mathcal{T} \mid T(x, y, v(z))=T(h(x), h(y), z)=0\}, \\
& \mathcal{W}_{4}=\{T \in \mathcal{T} \mid T(x, y, h(z))=T(h(x), h(y), z)=0\},
\end{aligned}
$$

where $x, y, z \in V$.

The torsion forms associated with $T \in \mathcal{T}$ are defined as follows:

$$
t(x)=g^{i j} T\left(x, e_{i}, e_{j}\right), \quad t^{*}(x)=g^{i j} T\left(x, e_{i}, \varphi e_{j}\right), \quad \hat{t}(x)=T(x, \xi, \xi)
$$

regarding the basis $\left\{e_{i} ; \xi\right\}(i=1,2, \ldots, 2 n)$ of $V$. Obviously, $\hat{t}(\xi)=0$ is always valid.

According to Corollary 2.5, (5) and (6) we obtain the following 
Corollary 2.6. The torsion forms of $T$ have the following properties in each of the subspaces $\mathcal{W}_{i}(i=1,2,3,4)$ :

(i) If $T \in \mathcal{W}_{1}$, then $t \circ v=t^{*} \circ v=\hat{t}=0$;

(ii) If $T \in \mathcal{W}_{2}$, then $t=t^{*}=\hat{t}=0$;

(iii) If $T \in \mathcal{W}_{3}$, then $t \circ h=t^{*} \circ h=\hat{t}=0$;

(iv) If $T \in \mathcal{W}_{4}$, then $t=t^{*}=0$.

Further we continue the decomposition of the subspaces $\mathcal{W}_{i}(i=1,2,3,4)$ of $\mathcal{T}$.

2.1. The subspace $\mathcal{W}_{1}$. Since the endomorphism $\varphi$ induces an almost complex structure on $H=\operatorname{ker}(\eta)$ (which is the orthogonal complement $\{\xi\}^{\perp}$ of the subspace $\operatorname{span}(\xi)$ ) and the restriction of $g$ on $H$ is a Norden metric (because the almost complex structure causes an anti-isometry on $H$ ), then the decomposition of $\mathcal{W}_{1}$ is made as the decomposition of the space of the torsion tensors on an almost complex manifold with Norden metric known from [10].

Let us consider the linear operator $L_{1,0}: \mathcal{W}_{1} \rightarrow \mathcal{W}_{1}$ defined by

$$
L_{1,0}(T)(x, y, z)=-T\left(\varphi x, \varphi y, \varphi^{2} z\right) .
$$

Then, it follows immediately

Lemma 2.7. The operator $L_{1,0}$ is an involutive isometry on $\mathcal{W}_{1}$ and it is invariant with respect to the group $\mathcal{G} \times \mathcal{I}$, i.e.

$$
L_{1,0} \circ L_{1,0}=\operatorname{Id}_{\mathcal{W}_{1}}, \quad\left\langle L_{1,0}\left(T_{1}\right), L_{1,0}\left(T_{2}\right)\right\rangle=\left\langle T_{1}, T_{2}\right\rangle, \quad L_{1,0}((\lambda a) T)=(\lambda a)\left(L_{1,0}(T)\right),
$$

where $T_{1}, T_{2} \in \mathcal{W}_{1}, a \in \mathcal{G} \times \mathcal{I}$.

Therefore, $L_{1,0}$ has two eigenvalues +1 and -1 , and the corresponding eigenspaces

$$
\mathcal{W}_{1}^{+}=\left\{T \in \mathcal{W}_{1} \mid L_{1,0}(T)=T\right\}, \quad \mathcal{W}_{1}^{-}=\left\{T \in \mathcal{W}_{1} \mid L_{1,0}(T)=-T\right\}
$$

are invariant orthogonal subspaces of $\mathcal{W}_{1}$. by

In order to decompose $\mathcal{W}_{1}^{-}$, we consider the linear operator $L_{1,1}: \mathcal{W}_{1}^{-} \rightarrow \mathcal{W}_{1}^{-}$defined

$$
L_{1,1}(T)(x, y, z)=-T\left(\varphi x, \varphi^{2} y, \varphi z\right) .
$$

Let us denote the eigenspaces $\mathcal{W}_{1,1 / 1,2}=\left\{T \in \mathcal{W}_{1}^{-} \mid L_{1,1}(T)=\mp T\right\}$. We have

Lemma 2.8. The operator $L_{1,1}$ is an involutive isometry on $\mathcal{W}_{1}$ and it is invariant with respect to $\mathcal{G} \times \mathcal{I}$.

According to the latter lemma, the eigenspaces $\mathcal{W}_{1,1}$ and $\mathcal{W}_{1,2}$ are invariant and orthogonal.

To decompose $\mathcal{W}_{1}^{+}$, we define the linear operator $L_{1,2}: \mathcal{W}_{1}^{+} \rightarrow \mathcal{W}_{1}^{+}$as follows:

$$
L_{1,2}(T)(x, y, z)=-\frac{1}{2}\left\{T\left(\varphi^{2} z, \varphi^{2} x, \varphi^{2} y\right)+T\left(\varphi^{2} z, \varphi x, \varphi y\right)\right\}_{[x \leftrightarrow y]} .
$$

Lemma 2.9. The operator $L_{1,2}$ is an involutive isometry on $\mathcal{W}_{1}^{+}$and it is invariant with respect to $\mathcal{G} \times \mathcal{I}$.

Thus, the eigenspaces $\mathcal{W}_{1,3 / 1,4}=\left\{T \in \mathcal{W}_{1}^{+} \mid L_{1,2}(T)= \pm T\right\}$ are invariant and orthogonal.

Using Lemma 2.7, Lemma 2.8 and Lemma 2.9, we get the following 
Theorem 2.10. The decomposition $\mathcal{W}_{1}=\mathcal{W}_{1,1} \oplus \mathcal{W}_{1,2} \oplus \mathcal{W}_{1,3} \oplus \mathcal{W}_{1,4}$ is orthogonal and invariant with respect to the structure group.

Bearing in mind the definition of the subspaces $\mathcal{W}_{1, i}(i=1,2,3,4)$, we obtain

Proposition 2.11. The subspaces $\mathcal{W}_{1, i}(i=1,2,3,4)$ of $\mathcal{W}_{1}$ are determined by:

$$
\begin{array}{lll}
\mathcal{W}_{1,1}: & T(\xi, y, z)=T(x, y, \xi)=0, & T(x, y, z)=-T(\varphi x, \varphi y, z)=-T(x, \varphi y, \varphi z) ; \\
\mathcal{W}_{1,2}: & T(\xi, y, z)=T(x, y, \xi)=0, & T(x, y, z)=-T(\varphi x, \varphi y, z)=T(\varphi x, y, \varphi z) ; \\
\mathcal{W}_{1,3}: & T(\xi, y, z)=T(x, y, \xi)=0, & T(x, y, z)-T(\varphi x, \varphi y, z)=\underset{x, y, z}{\mathfrak{S}} T(x, y, z)=0 ; \\
\mathcal{W}_{1,4}: & T(\xi, y, z)=T(x, y, \xi)=0, & T(x, y, z)-T(\varphi x, \varphi y, z)=\underset{x, y, z}{\mathfrak{S}} T(\varphi x, y, z)=0 .
\end{array}
$$

Using Corollary 2.6 (i), Proposition 2.11 and (6), we obtain

Corollary 2.12. The torsion forms $t$ and $t^{*}$ of $T$ have the following properties in the subspaces $\mathcal{W}_{1, i}(i=1,2,3,4)$ :

(i) If $T \in \mathcal{W}_{1,1}$, then $t=-t^{*} \circ \varphi, t \circ \varphi=t^{*} ; \quad$ (ii) If $T \in \mathcal{W}_{1,2}$, then $t=t^{*}=0$;

(iii) If $T \in \mathcal{W}_{1,3}$, then $t=t^{*} \circ \varphi, t \circ \varphi=-t^{*} ; \quad$ (iv) If $T \in \mathcal{W}_{1,4}$, then $t=t^{*}=0$.

Let us remark that each of the subspaces $\mathcal{W}_{1,1}$ and $\mathcal{W}_{1,3}$ can be additionally decomposed to a couple of subspaces - one of zero traces $\left(t, t^{*}\right)$ and one of non-zero traces $\left(t, t^{*}\right)$, i.e.

$$
\mathcal{W}_{1,1}=\mathcal{W}_{1,1,1} \oplus \mathcal{W}_{1,1,2}, \quad \mathcal{W}_{1,3}=\mathcal{W}_{1,3,1} \oplus \mathcal{W}_{1,3,2}
$$

where

$$
\begin{array}{ll}
\mathcal{W}_{1,1,1}=\left\{T \in \mathcal{W}_{1,1} \mid t \neq 0\right\}, & \mathcal{W}_{1,3,1}=\left\{T \in \mathcal{W}_{1,3} \mid t \neq 0\right\}, \\
\mathcal{W}_{1,1,2}=\left\{T \in \mathcal{W}_{1,1} \mid t=0\right\}, & \mathcal{W}_{1,3,2}=\left\{T \in \mathcal{W}_{1,3} \mid t=0\right\} .
\end{array}
$$

Proposition 2.13. Let $T \in \mathcal{T}$ and $p_{1, i}(i=1,2,3,4)$ be the projection operators of $\mathcal{T}$ in $\mathcal{W}_{1, i}$, generated by the decomposition above. Then we have

$$
\begin{aligned}
p_{1,1 / 1,2}(T)(x, y, z)= & -\frac{1}{4}\left\{T\left(\varphi^{2} x, \varphi^{2} y, \varphi^{2} z\right)-T\left(\varphi x, \varphi y, \varphi^{2} z\right) \mp T\left(\varphi x, \varphi^{2} y, \varphi z\right)\right. \\
& \left.\mp T\left(\varphi^{2} x, \varphi y, \varphi z\right)\right\} \\
p_{1,3 / 1,4}(T)(x, y, z)= & -\frac{1}{4}\left\{T\left(\varphi^{2} x, \varphi^{2} y, \varphi^{2} z\right)+T\left(\varphi x, \varphi y, \varphi^{2} z\right)\right\} \pm \frac{1}{8}\left\{T\left(\varphi^{2} z, \varphi^{2} x, \varphi^{2} y\right)\right. \\
& \left.+T\left(\varphi^{2} z, \varphi x, \varphi y\right)+T\left(\varphi z, \varphi x, \varphi^{2} y\right)-T\left(\varphi z, \varphi^{2} x, \varphi y\right)\right\}_{[x \leftrightarrow y]} .
\end{aligned}
$$

Proof. Let us show the calculations about $p_{1,1}$ for example, using [10]. Lemma 2.7 implies that the tensor $\frac{1}{2}\left\{T-L_{1,0}(T)\right\}$ is the projection of $T \in \mathcal{W}_{1}$ in $\mathcal{W}_{1}^{-}=\mathcal{W}_{1,1} \oplus \mathcal{W}_{1,2}$. Using Lemma 2.8. we find the expression of $p_{1,1}$ in terms of the operators $L_{1,0}$ and $L_{1,1}$ for $T \in \mathcal{W}_{1}$, namely

$$
p_{1,1}(T)=\frac{1}{4}\left\{T-L_{1,0}(T)-L_{1,1}(T)+L_{1,1} \circ L_{1,0}(T)\right\},
$$

which implies the stated expression of $p_{1,1}$, taking into account that $T \in \mathcal{W}_{1}$ is the image of $T \in \mathcal{T}$ by $p_{1}$. In a similar way we prove the expressions for the other projectors under consideration.

We verify that $p_{1, i} \circ p_{1, i}=p_{1, i}$ and $\sum_{i} p_{1, i}=\operatorname{Id}_{\mathcal{W}_{1}}$ for $i=1,2,3,4$. 
2.2. The subspace $\mathcal{W}_{2}$. Following the demonstrated procedure for $\mathcal{W}_{1}$, we continue the decomposition of the other main subspaces of $\mathcal{T}$ with respect to the almost contact B-metric structure.

Lemma 2.14. The operator $L_{2,0}$, defined by $L_{2,0}(T)(x, y, z)=\eta(z) T(\varphi x, \varphi y, \xi)$, is an involutive isometry on $\mathcal{W}_{2}$ and invariant with respect to $\mathcal{G} \times \mathcal{I}$.

Hence, the corresponding eigenspaces $\mathcal{W}_{2,1 / 2,2}=\left\{T \in \mathcal{W}_{2} \mid L_{2,0}(T)=\mp T\right\}$ are invariant and orthogonal. Therefore, we have

Theorem 2.15. The decomposition $\mathcal{W}_{2}=\mathcal{W}_{2,1} \oplus \mathcal{W}_{2,2}$ is orthogonal and invariant with respect to the structure group.

Proposition 2.16. The subspaces of $\mathcal{W}_{2}$ are determined by:

$$
\mathcal{W}_{2,1 / 2,2}: \quad T(x, y, z)=\eta(z) T\left(\varphi^{2} x, \varphi^{2} y, \xi\right), \quad T(x, y, \xi)=\mp T(\varphi x, \varphi y, \xi) .
$$

Then the tensors $\frac{1}{2}\left\{T-L_{2,0}(T)\right\}$ and $\frac{1}{2}\left\{T+L_{2,0}(T)\right\}$ are the projections of $\mathcal{W}_{2}$ in $\mathcal{W}_{2,1}$ and $\mathcal{W}_{2,2}$, respectively. Moreover, we have $p_{2, j} \circ p_{2, j}=p_{2, j}(j=1,2)$ and $p_{2,1}+p_{2,2}=$ $\operatorname{Id}_{\mathcal{W}_{2}}$. Therefore, taking into account $p_{2}$, we obtain

Proposition 2.17. Let $T \in \mathcal{T}$ and $p_{2, j}(j=1,2)$ be the projection operators of $\mathcal{T}$ in $\mathcal{W}_{2, j}$, generated by the decomposition above. Then we have

$$
p_{2,1 / 2,2}(T)(x, y, z)=\frac{1}{2} \eta(z)\left\{T\left(\varphi^{2} x, \varphi^{2} y, \xi\right) \mp T(\varphi x, \varphi y, \xi)\right\} .
$$

According to Corollary 2.6 (ii), Proposition 2.16 and (6) we obtain the following

Corollary 2.18. The torsion forms of $T$ are zero in each of the subspaces $\mathcal{W}_{2,1}$ and $\mathcal{W}_{2,2}$, i.e. if $T \in \mathcal{W}_{2,1} \oplus \mathcal{W}_{2,2}$, then $t=t^{*}=\hat{t}=0$.

\subsection{The subspace $\mathcal{W}_{3}$.}

Lemma 2.19. The following operators $L_{3, k}(k=0,1)$ are involutive isometries on $\mathcal{W}_{3}$ and invariant with respect to $\mathcal{G} \times \mathcal{I}$ :

$$
\begin{aligned}
& L_{3,0}(T)(x, y, z)=\{\eta(x) T(\xi, \varphi y, \varphi z)\}_{[x \leftrightarrow y]}, \\
& L_{3,1}(T)(x, y, z)=\left\{\eta(x) T\left(\xi, \varphi^{2} z, \varphi^{2} y\right)\right\}_{[x \leftrightarrow y]} .
\end{aligned}
$$

By virtue of their action, we obtain consecutively the corresponding invariant and orthogonal eigenspaces:

$$
\begin{aligned}
\mathcal{W}_{3}^{-}=\left\{T \in \mathcal{W}_{3} \mid L_{3,0}(T)=-T\right\}, & \mathcal{W}_{3}^{+}=\left\{T \in \mathcal{W}_{3} \mid L_{3,0}(T)=T\right\}, \\
\mathcal{W}_{3,1 / 3,2}=\left\{T \in \mathcal{W}_{3}^{-} \mid L_{3,1}(T)= \pm T\right\}, & \mathcal{W}_{3,3 / 3,4}=\left\{T \in \mathcal{W}_{3}^{+} \mid L_{3,1}(T)= \pm T\right\} .
\end{aligned}
$$

In such a way, we get

Theorem 2.20. The decomposition $\mathcal{W}_{3}=\mathcal{W}_{3,1} \oplus \mathcal{W}_{3,2} \oplus \mathcal{W}_{3,3} \oplus \mathcal{W}_{3,4}$ is orthogonal and invariant with respect to the structure group. 
Proposition 2.21. The subspaces of $\mathcal{W}_{3}$ are determined by:

$$
\begin{aligned}
\mathcal{W}_{3,1 / 3,2}: & T(x, y, z)=\left\{\eta(x) T\left(\xi, \varphi^{2} y, \varphi^{2} z\right)\right\}_{[x \leftrightarrow y]}, \\
& T(\xi, y, z)= \pm T(\xi, z, y)=-T(\xi, \varphi y, \varphi z) \\
\mathcal{W}_{3,3 / 3,4}: & T(x, y, z)=\left\{\eta(x) T\left(\xi, \varphi^{2} y, \varphi^{2} z\right)\right\}_{[x \leftrightarrow y]}, \\
& T(\xi, y, z)= \pm T(\xi, z, y)=T(\xi, \varphi y, \varphi z) .
\end{aligned}
$$

By virtue of Corollary 2.6 (iii), Proposition 2.21 and (6) we obtain

Corollary 2.22. The torsion forms $t$ and $t^{*}$ of $T$ are zero in $\mathcal{W}_{3, k} \subset \mathcal{W}_{3}(k=2,3,4)$.

Let us remark that $\mathcal{W}_{3,1}$ can be additionally decomposed to three subspaces determined by conditions $t=0, t^{*}=0$ and $t=t^{*}=0$, respectively, i.e.

$$
\mathcal{W}_{3,1}=\mathcal{W}_{3,1,1} \oplus \mathcal{W}_{3,1,2} \oplus \mathcal{W}_{3,1,3},
$$

where

$$
\begin{gathered}
\mathcal{W}_{3,1,1}=\left\{T \in \mathcal{W}_{3,1} \mid t \neq 0, t^{*}=0\right\}, \quad \mathcal{W}_{3,1,2}=\left\{T \in \mathcal{W}_{3,1} \mid t=0, t^{*} \neq 0\right\}, \\
\mathcal{W}_{3,1,3}=\left\{T \in \mathcal{W}_{3,1} \mid t=0, t^{*}=0\right\} .
\end{gathered}
$$

Proposition 2.23. Let $T \in \mathcal{T}$ and $p_{3, k}(k=1,2,3,4)$ be the projection operators of $\mathcal{T}$ in $\mathcal{W}_{3, k}$, generated by the decomposition above. Then we have

$$
p_{3, k}(T)(x, y, z)=\frac{1}{4}\left\{\eta(x) A_{3, k}(y, z)-\eta(y) A_{3, k}(x, z)\right\},
$$

where

$$
\begin{aligned}
& A_{3,1 / 3,2}(y, z)=T\left(\xi, \varphi^{2} y, \varphi^{2} z\right) \pm T\left(\xi, \varphi^{2} z, \varphi^{2} y\right)-T(\xi, \varphi y, \varphi z) \mp T(\xi, \varphi z, \varphi y), \\
& A_{3,3 / 3,4}(y, z)=T\left(\xi, \varphi^{2} y, \varphi^{2} z\right) \pm T\left(\xi, \varphi^{2} z, \varphi^{2} y\right)+T(\xi, \varphi y, \varphi z) \pm T(\xi, \varphi z, \varphi y) .
\end{aligned}
$$

2.4. The subspace $\mathcal{W}_{4}$. Finally, we only denote $\mathcal{W}_{4}$ as $\mathcal{W}_{4,1}$ and it is determined as follows

$$
\mathcal{W}_{4,1}: \quad T(x, y, z)=\eta(z)\{\eta(y) \hat{t}(x)-\eta(x) \hat{t}(y)\} .
$$

Obviously, the projection operator $p_{4,1}: \mathcal{T} \rightarrow \mathcal{W}_{4,1}$ has the form

$$
p_{4,1}(T)(x, y, z)=\eta(z)\{\eta(y) \hat{t}(x)-\eta(x) \hat{t}(y)\} .
$$

2.5. The fifteen subspaces of $\mathcal{T}$. In conclusion of the decomposition explained above, we combine Theorems 2.4, 2.10, 2.15] and 2.20. We denote the subspaces $\mathcal{W}_{i, j}$ and $\mathcal{W}_{i, j, k}$ by $\mathcal{T}_{s}, s \in\{1,2, \ldots, 15\}$ as follows:

$$
\begin{array}{lllll}
\mathcal{T}_{1}=\mathcal{W}_{1,1,1}, & \mathcal{T}_{2}=\mathcal{W}_{1,1,2}, & \mathcal{T}_{3}=\mathcal{W}_{1,2}, & \mathcal{T}_{4}=\mathcal{W}_{1,3,1}, & \mathcal{T}_{5}=\mathcal{W}_{1,3,2}, \\
\mathcal{T}_{6}=\mathcal{W}_{1,4}, & \mathcal{T}_{7}=\mathcal{W}_{2,1}, & \mathcal{T}_{8}=\mathcal{W}_{2,2}, & \mathcal{T}_{9}=\mathcal{W}_{3,1,1}, & \mathcal{T}_{10}=\mathcal{W}_{3,1,2}, \\
\mathcal{T}_{11}=\mathcal{W}_{3,1,3}, & \mathcal{T}_{12}=\mathcal{W}_{3,2}, & \mathcal{T}_{13}=\mathcal{W}_{3,3}, & \mathcal{T}_{14}=\mathcal{W}_{3,4}, & \mathcal{T}_{15}=\mathcal{W}_{4,1}
\end{array}
$$

We obtain the following main statement in the present paper 
Theorem 2.24. Let $\mathcal{T}$ is the vector space of the torsion tensors of type (0,3) over the vector space $V$ with almost contact $B$-matric structure $(\varphi, \xi, \eta, g)$. The decomposition

$$
\mathcal{T}=\mathcal{T}_{1} \oplus \mathcal{T}_{2} \oplus \cdots \oplus \mathcal{T}_{15}
$$

is orthogonal and invariant with respect to the structure group $\mathcal{G} \times \mathcal{I}$.

In the following section we discuss three known natural connections with torsion on $(M, \varphi, \xi, \eta, g)$. Natural connections are a generalization of the Levi-Civita connection.

\section{Known Natural Connections in the Introduced Classification}

Let $(M, \varphi, \xi, \eta, g)$ be an almost contact B-metric manifold. The tangent space $T_{p} M$ at an arbitrary point $p$ in $M$ is a vector space equipped with an almost contact B-metric structure.

It is well known that any metric connection $D$ (i.e. $D g=0$ ) is completely determined by its torsion tensor $T$ with

$$
2 g\left(D_{x} y-\nabla_{x} y, z\right)=T(x, y, z)-T(y, z, x)+T(z, x, y) .
$$

Then the subspace $\mathcal{T}_{s}(s=1,2, \ldots, 15)$, where $T$ belongs, is an important characteristic of $D$. In such a way the conditions for $T$ described as the subspace $\mathcal{T}_{s}$ give rise to the corresponding class of the connection with respect to its torsion tensor.

A metric connection $D$ is called a natural connection on $(M, \varphi, \xi, \eta, g)$ if the almost contact structure $(\varphi, \xi, \eta)$ as well as the B-metric $g$ (consequently also $\widetilde{g}$ ) are parallel regarding it, i.e. $D \varphi=D \xi=D \eta=D g=D \widetilde{g}=0$. Therefore, an arbitrary natural connection $D$ on $(M, \varphi, \xi, \eta, g) \notin \mathcal{F}_{0}$ plays the same role like $\nabla$ on $(M, \varphi, \xi, \eta, g) \in \mathcal{F}_{0}$. Obviously, $D$ and $\nabla$ coincide when $(M, \varphi, \xi, \eta, g) \in \mathcal{F}_{0}$. Because of that, we are interested in natural connections on $(M, \varphi, \xi, \eta, g) \notin \mathcal{F}_{0}$.

Theorem 3.1. A linear connection $D$ is natural on $(M, \varphi, \xi, \eta, g)$ if and only if $D \varphi=$ $D g=0$.

Proof. It is known, that a linear connection $D$ is a natural connection on $(M, \varphi, \xi, \eta, g)$ if and only if the following properties for $Q(x, y, z)=g\left(D_{x} y-\nabla_{x} y, z\right)$ are valid [24]:

$$
Q(x, y, \varphi z)-Q(x, \varphi y, z)=F(x, y, z), \quad Q(x, y, z)=-Q(x, z, y) .
$$

These conditions are equivalent to $D \varphi=0$ and $D g=0$, respectively. Moreover, $D \xi=0$ is equivalent to the relation $Q(x, \xi, z)=-F(x, \xi, \varphi z)$, which is a consequence of the former equality of (12). Finally, since $\eta(\cdot)=g(\cdot, \xi)$, then supposing $D g=0$ we have $D \xi=0$ if and only if $D \eta=0$. Thus, the statement is truthful.

Proposition 3.2. Let $D$ be a natural connection with torsion $T$ on an almost contact $B$-metric manifold $M$. Then the following implications hold true:

$$
\begin{array}{lll} 
& T \in \mathcal{T}_{1} \oplus \mathcal{T}_{2} \oplus \mathcal{T}_{6} \oplus \mathcal{T}_{12} \Rightarrow M \in \mathcal{F}_{0} ; & \\
T \in \mathcal{T}_{3} \Rightarrow M \in \mathcal{F}_{3} ; & T \in \mathcal{T}_{4} \Rightarrow M \in \mathcal{F}_{1} ; & T \in \mathcal{T}_{5} \Rightarrow M \in \mathcal{F}_{2} ; \\
T \in \mathcal{T}_{7} \Rightarrow M \in \mathcal{F}_{7} ; & T \in \mathcal{T}_{8} \Rightarrow M \in \mathcal{F}_{8} \oplus \mathcal{F}_{10} ; & T \in \mathcal{T}_{9} \Rightarrow M \in \mathcal{F}_{5} ; \\
T \in \mathcal{T}_{10} \Rightarrow M \in \mathcal{F}_{4} ; & T \in \mathcal{T}_{11} \Rightarrow M \in \mathcal{F}_{6} ; & T \in \mathcal{T}_{13} \Rightarrow M \in \mathcal{F}_{9} ; \\
T \in \mathcal{T}_{14} \Rightarrow M \in \mathcal{F}_{10} ; & T \in \mathcal{T}_{15} \Rightarrow M \in \mathcal{F}_{11} . &
\end{array}
$$


Proof. The implications follow from (11), (12), (11), (9) and the corresponding characteristic conditions of $\mathcal{W}_{i, j}$ and $\mathcal{W}_{i, j, k}$ as well as the projection operators $p_{i, j}$. We show the proof in detail for some classes and the rest follow in a similar way.

By virtue of (11) and (12) we have

$$
\begin{aligned}
2 F(x, y, z) & =T(x, y, \varphi z)-T(y, \varphi z, x)+T(\varphi z, x, y) \\
& -T(x, \varphi y, z)+T(\varphi y, z, x)-T(z, x, \varphi y) .
\end{aligned}
$$

Let us consider $T \in \mathcal{W}_{1,1}=\mathcal{T}_{1} \oplus \mathcal{T}_{2}$, which is equivalent to $T=p_{1,1}(T)$. Then, according to Proposition 2.13, we have

$$
\begin{array}{r}
T(x, y, z)=-\frac{1}{4}\left\{T\left(\varphi^{2} x, \varphi^{2} y, \varphi^{2} z\right)-T\left(\varphi x, \varphi y, \varphi^{2} z\right)\right. \\
\left.-T\left(\varphi x, \varphi^{2} y, \varphi z\right)-T\left(\varphi^{2} x, \varphi y, \varphi z\right)\right\},
\end{array}
$$

which together with (13) imply $F(x, y, z)=0$. Therefore, we obtain $M \in \mathcal{F}_{0}$.

Now, let us suppose $T \in \mathcal{W}_{1,2}=\mathcal{T}_{3}$ and hence $T=p_{1,2}(T)$, which has the following form, taking into account Proposition 2.13;

$$
\begin{aligned}
T(x, y, z)=-\frac{1}{4}\{ & T\left(\varphi^{2} x, \varphi^{2} y, \varphi^{2} z\right)-T\left(\varphi x, \varphi y, \varphi^{2} z\right) \\
+ & \left.T\left(\varphi x, \varphi^{2} y, \varphi z\right)+T\left(\varphi^{2} x, \varphi y, \varphi z\right)\right\} .
\end{aligned}
$$

Then, according to the latter equality and (13), we obtain

$$
\begin{aligned}
F(x, y, z)=-\frac{1}{4}\{ & -T\left(\varphi^{2} x, \varphi^{2} y, \varphi z\right)+T(\varphi x, \varphi y, \varphi z)+T\left(\varphi^{2} x, \varphi y, \varphi^{2} z\right) \\
& +T\left(\varphi x, \varphi^{2} y, \varphi^{2} z\right)-T\left(\varphi z, \varphi^{2} x, \varphi^{2} y\right)-T\left(\varphi^{2} z, \varphi x, \varphi^{2} y\right) \\
& \left.+T\left(\varphi^{2} z, \varphi^{2} x, \varphi y\right)-T(\varphi z, \varphi x, \varphi y)\right\}
\end{aligned}
$$

and consequently $F(\xi, y, z)=F(x, y, \xi)=0$. Next, we take the cyclic sum of (14) by the arguments $x, y, z$ and the result is $\underset{x, y, z}{\mathfrak{S}} F(x, y, z)=0$. Therefore, $M$ belongs to $\mathcal{F}_{3}$.

Bearing in mind the class of almost contact B-metric manifolds with $N=0$ and Proposition 3.2, we obtain immediately

Corollary 3.3. An almost contact B-metric manifold $M=(M, \varphi, \xi, \eta, g) \in \mathcal{F}_{i} \backslash \mathcal{F}_{0}$ is normal, i.e. $N=0$, if the torsion of an arbitrary natural connection on $M$ belongs to $\mathcal{T}_{4} \oplus \mathcal{T}_{5} \oplus \mathcal{T}_{9} \oplus \mathcal{T}_{10} \oplus \mathcal{T}_{11}$

Similarly, Proposition 1.2 and Proposition 3.2 imply

Corollary 3.4. An almost contact B-metric manifold $M=(M, \varphi, \xi, \eta, g) \in \mathcal{F}_{i} \backslash \mathcal{F}_{0}$ has $\widehat{N}=0$, if the torsion of an arbitrary natural connection on $M$ belongs to $\mathcal{T}_{3} \oplus \mathcal{T}_{7}$.

3.1. The $\varphi$ B-connection in the classification. In [25], it is introduced a natural connection $\dot{D}$ on $(M, \varphi, \xi, \eta, g)$ in any basic class by

$$
\dot{D}_{x} y=\nabla_{x} y+\frac{1}{2}\left\{\left(\nabla_{x} \varphi\right) \varphi y+\left(\nabla_{x} \eta\right) y \cdot \xi\right\}-\eta(y) \nabla_{x} \xi
$$

In [26], this connection is called a $\varphi$ B-connection. It is studied for some classes of the manifolds $(M, \varphi, \xi, \eta, g)$ in [25, 22, 23, 26]. The $\varphi$ B-connection is the odd-dimensional analogue of the B-connection on the corresponding almost complex manifold with Norden metric, studied in [8] for the class of the conformal Kähler manifold with Norden metric. 
This connection has a torsion tensor and torsion 1-forms as follows:

$$
\begin{gathered}
\dot{T}(x, y, z)=\left\{-\frac{1}{2} F\left(x, \varphi y, \varphi^{2} z\right)+\eta(x) F(y, \varphi z, \xi)+\eta(z) F(x, \varphi y, \xi)\right\}_{[x \leftrightarrow y]}, \\
\dot{t}=\frac{1}{2}\left\{\theta^{*}+\theta^{*}(\xi) \eta\right\}, \quad \dot{t}^{*}=-\frac{1}{2}\{\theta+\theta(\xi) \eta\}, \quad \hat{\dot{t}}=-\omega \circ \varphi .
\end{gathered}
$$

Applying Propositions 2.13, 2.17, 2.23 and equation (8) for the torsion tensor $\dot{T}$ from (15), we obtain the components of $\dot{T}$ in each of the subspaces $\mathcal{W}_{i, j}$ :

$$
\begin{aligned}
& p_{1,1}(\dot{T})(x, y, z)=0 \text {, } \\
& p_{1,2}(\dot{T})(x, y, z)=-\frac{1}{4}\left\{F\left(\varphi^{2} x, \varphi^{2} y, \varphi z\right)-F(\varphi x, \varphi y, \varphi z)\right\}_{[x \leftrightarrow y]}, \\
& p_{1,3 / 1,4}(\dot{T})(x, y, z)=-\frac{1}{8}\left\{F\left(\varphi^{2} z, \varphi^{2} y, \varphi x\right) \pm F\left(\varphi^{2} x, \varphi^{2} y, \varphi z\right)\right. \\
& \pm F(\varphi x, \varphi y, \varphi z)\}_{[x \leftrightarrow y]}, \\
& p_{2,1 / 2,2}(\dot{T})(x, y, z)=-\frac{1}{2} \eta(z)\left\{F\left(\varphi^{2} x, \varphi y, \xi\right) \mp F(\varphi x, y, \xi)\right\}_{[x \leftrightarrow y]}, \\
& p_{3,1 / 3,2}(\dot{T})(x, y, z)=\frac{1}{4}\left\{\eta ( y ) \left[F\left(\varphi^{2} x, \varphi z, \xi\right) \pm F\left(\varphi^{2} z, \varphi x, \xi\right)\right.\right. \\
& -F(\varphi x, z, \xi) \mp F(\varphi z, x, \xi)]\}_{[x \leftrightarrow y]}, \\
& p_{3,3}(\dot{T})(x, y, z)=\frac{1}{4}\left\{\eta ( y ) \left[F\left(\varphi^{2} x, \varphi z, \xi\right)+F\left(\varphi^{2} z, \varphi x, \xi\right)\right.\right. \\
& +F(\varphi x, z, \xi)+F(\varphi z, x, \xi)]\}_{[x \leftrightarrow y]}, \\
& p_{3,4}(\dot{T})(x, y, z)=\frac{1}{4}\left\{\eta ( y ) \left[F\left(\varphi^{2} x, \varphi z, \xi\right)-F\left(\varphi^{2} z, \varphi x, \xi\right)+F(\varphi x, z, \xi)\right.\right. \\
& \left.\left.-F(\varphi z, x, \xi)+2 F\left(\xi, \varphi x, \varphi^{2} z\right)\right]\right\}_{[x \leftrightarrow y]}, \\
& p_{4,1}(\dot{T})(x, y, z)=\eta(z)\{\eta(x) \omega(\varphi y)-\eta(y) \omega(\varphi x)\} .
\end{aligned}
$$

Such a way we establish the position of the torsion of $\dot{D}$ in the classification (10) as follows

Proposition 3.5. The torsion $\dot{T}$ of the $\varphi$ B-connection on $(M, \varphi, \xi, \eta, g)$ belongs to $\mathcal{T}_{3} \oplus$ $\mathcal{T}_{4} \oplus \cdots \oplus \mathcal{T}_{15}$.

3.2. The $\varphi \mathbf{K T}$-connection in the classification. In 24, it is introduced a natural connection on $(M, \varphi, \xi, \eta, g)$, called a $\varphi K T$-connection, which torsion tensor $\ddot{T}$ is totally skew-symmetric, i.e. a 3 -form. The $\varphi \mathrm{KT}$-connection is the odd-dimensional analogue of the KT-connection introduced in [29] on the corresponding class of quasi-Kähler almost complex manifolds with Norden metric.

Corollary 3.6. The $\varphi K T$-connection exists on an almost contact B-metric manifold $(M, \varphi, \xi, \eta, g)$ if and only if the tensor $\widehat{N}$ vanishes on it.

Proof. It is proved in [24] that $\varphi \mathrm{KT}$-connection exists only on $(M, \varphi, \xi, \eta, g) \in \mathcal{F}_{3} \oplus \mathcal{F}_{7}$, i.e. the class of almost contact B-metric manifolds, where $\xi$ is a Killing vector field and the cyclic sum $\mathfrak{S}$ of $F$ by three arguments is zero. According to Proposition 1.2, the class $\mathcal{F}_{3} \oplus \mathcal{F}_{7}$ is characterized by the condition $\widehat{N}=0$ which completes the proof.

The unique $\varphi \mathrm{KT}$-connection $\ddot{D}$ is determined by

$$
g\left(\ddot{D}_{x} y, z\right)=g\left(\nabla_{x} y, z\right)+\frac{1}{2} \ddot{T}(x, y, z),
$$


where the torsion tensor is defined by

$$
\begin{aligned}
\ddot{T}(x, y, z) & =-\frac{1}{2} \underset{x, y, z}{\mathfrak{S}}\{F(x, y, \varphi z)-3 \eta(x) F(y, \varphi z, \xi)\} \\
& =(\eta \wedge \mathrm{d} \eta)(x, y, z)+\frac{1}{4} \underset{x, y, z}{\mathfrak{S}} N(x, y, z) .
\end{aligned}
$$

Obviously, the torsion forms of the $\varphi \mathrm{KT}$-connection are zero.

From (18), in a similar way of (17), we get the following non-zero components of $\ddot{T}$ :

$$
\begin{aligned}
p_{1,2}(\ddot{T})(x, y, z)=-\frac{1}{2}\{F(x, y, \varphi z)+F(y, z, \varphi x)-F(z, x, \varphi y) & \\
& \quad-\eta(x) F(y, \varphi z, \xi)+\eta(y) F(z, \varphi x, \xi)+\eta(z) F(x, \varphi y, \xi)\}, \\
p_{1,4}(\ddot{T})(x, y, z)=-F(z, x, \varphi y)-\eta(x) F(y, \varphi z, \xi), & \\
p_{2,1}(\ddot{T})(x, y, z)= & 2 \eta(z) F(x, \varphi y, \xi), \\
p_{3,2}(\ddot{T})(x, y, z)= & 2 \eta(x) F(y, \varphi z, \xi)+2 \eta(y) F(z, \varphi x, \xi) .
\end{aligned}
$$

Therefore we have

Proposition 3.7. The torsion $\ddot{T}$ of the $\varphi K T$-connection on $(M, \varphi, \xi, \eta, g) \in \mathcal{F}_{3} \oplus \mathcal{F}_{7}$ belongs to $\mathcal{T}_{3} \oplus \mathcal{T}_{6} \oplus \mathcal{T}_{7} \oplus \mathcal{T}_{12}$.

3.3. The $\varphi$-canonical connection in the classification. In [27], it is introduced a natural connection $\dddot{D}$ on $(M, \varphi, \xi, \eta, g)$, called a $\varphi$-canonical connection, if the torsion tensor $\dddot{T}$ of $\dddot{D}$ satisfies the following identity:

$$
\begin{gathered}
\{\dddot{T}(x, y, z)-\dddot{T}(x, \varphi y, \varphi z)-\eta(x)\{\dddot{T}(\xi, y, z)-\dddot{T}(\xi, \varphi y, \varphi z)\} \\
-\eta(y)\{\dddot{T}(x, \xi, z)-\dddot{T}(x, z, \xi)-\eta(x) \dddot{T}(z, \xi, \xi)\}\}_{[y \leftrightarrow z]}=0 .
\end{gathered}
$$

Let us remark that the restriction the $\varphi$-canonical connection of $(M, \varphi, \xi, \eta, g)$ on the contact distribution $\operatorname{ker}(\eta)$ is the unique canonical connection of the corresponding almost complex manifold with Norden metric, studied in [10].

The torsion tensor of the the $\varphi$-canonical connection is

$$
\dddot{T}(x, y, z)=\dot{T}(x, y, z)-\frac{1}{8}\left\{N\left(\varphi^{2} z, \varphi^{2} y, \varphi^{2} x\right)+2 N(\varphi z, \varphi y, \xi) \eta(x)\right\}_{[x \leftrightarrow y]},
$$

where $\dot{T}$ is the torsion tensor of the $\varphi \mathrm{B}$-connection from (15). The torsion forms are the same as in (16).

In [27, it is proved that the $\varphi \mathrm{B}$-connection and the $\varphi$-canonical connection of the manifold $(M, \varphi, \xi, \eta, g)$ coincide if and only if $N(\varphi \cdot \varphi \cdot)=0$, i.e. on any manifold from $\mathcal{F}_{i}, i \in\{1,2, \ldots, 11\} \backslash\{3,7\}$, where the $\varphi \mathrm{KT}$-connection does not exist. For the rest basic classes, where the $\varphi \mathrm{KT}$-connection exists, we obtain

Proposition 3.8. Let $(M, \varphi, \xi, \eta, g)$ be an arbitrary manifold in $\mathcal{F}_{i}, i \in\{3,7\}$. The $\varphi B$ connection $\dot{D}$ is the average connection of the $\varphi K T$-connection $\ddot{D}$ and the $\varphi$-canonical connection $\dddot{D}$, i.e. $2 \dot{D}=\ddot{D}+\dddot{D}$.

Proof. By virtue of (17), (19) and (21) we obtain: 
1) for $\mathcal{F}_{3}$

$$
\begin{aligned}
p_{1,2}(\dot{T})(x, y, z) & =p_{1,2}(\ddot{T})(x, y, z)=p_{1,2}(\dddot{T})(x, y, z) \\
& =-\frac{1}{2}\left\{F\left(\varphi^{2} x, \varphi^{2} y, \varphi z\right)+F\left(\varphi^{2} y, \varphi^{2} z, \varphi x\right)-F\left(\varphi^{2} z, \varphi^{2} x, \varphi y\right)\right\}, \\
2 p_{1,4}(\dot{T})(x, y, z) & =p_{1,4}(\ddot{T})(x, y, z)=-F\left(\varphi^{2} z, \varphi^{2} x, \varphi y\right), \quad p_{1,4}(\dddot{T})(x, y, z)=0
\end{aligned}
$$

2) for $\mathcal{F}_{7}$

$$
\begin{aligned}
p_{2,1}(\dot{T})(x, y, z) & =p_{2,1}(\ddot{T})(x, y, z)=p_{2,1}(\dddot{T})(x, y, z)=2 \eta(z) F(x, \varphi y, \xi), \\
2 p_{3,2}(\dot{T})(x, y, z) & =p_{3,2}(\ddot{T})(x, y, z)=2\{\eta(x) F(y, \varphi z, \xi)-\eta(y) F(x, \varphi z, \xi)\}, \\
p_{3,2}(\dddot{T})(x, y, z) & =0 .
\end{aligned}
$$

Therefore, we establish that $2 \dot{T}=\ddot{T}+\dddot{T}$ for $\mathcal{F}_{3}$ and $\mathcal{F}_{7}$. Then, using (11), we obtain $2 \dot{Q}=\ddot{Q}+\dddot{Q}$ for the corresponding tensors $\dot{Q}(x, y, z)=g\left(\dot{D}_{x} y-\nabla_{x} y, z\right), \ddot{Q}(x, y, z)=$ $g\left(\ddot{D}_{x} y-\nabla_{x} y, z\right), \dddot{Q}(x, y, z)=g\left(\dddot{D}_{x} y-\nabla_{x} y, z\right)$. Therefore, we have the statement.

Proposition 3.2 and Proposition 3.8 imply

Corollary 3.9. The torsion of the $\varphi$-canonical connection on $(M, \varphi, \xi, \eta, g)$ belongs to $\mathcal{T}_{3}$ and $\mathcal{T}_{7}$ if and only if $(M, \varphi, \xi, \eta, g)$ belongs to $\mathcal{F}_{3}$ and $\mathcal{F}_{7}$, respectively.

Remark 3.10. The implications in Proposition 3.2 become equivalences for the $\varphi$-canonical connection on $(M, \varphi, \xi, \eta, g) \in \mathcal{F}_{i}, i \in\{1,2, \ldots, 11\} \backslash\{3,7\}$, according to [27].

\section{ACKNOWLEDGMENTS}

The authors wish to thank Stefan Ivanov for his useful advices about this work. This work was financially supported by the Scientific Research Fund, Paisii Hilendarski University of Plovdiv, Bulgaria and the German Academic Exchange Service (DAAD).

\section{REFERENCES}

[1] Alexiev V., Ganchev G., On the classification of almost contact metric manifolds, In: Math. and Educ in Math., Proc. of 15th Spring Conf. of UBM, Sunny Beach, 1986, 155-161, (arXiv:1110.4297)

[2] Bismut J.-M., A local index theorem for non-Kähler manifolds, Math. Ann., 1989, 284, 681-699

[3] Biquard O., Métriques d'Einstein asymptotiquement symétriques, Astérisque, 2000, 265; English translation: Asymptotically Symmetric Einstein Metrics, SMF/AMS Texts and Monographs, American Mathematical Soc., 2006, 13

[4] Chern S.S., Complex manifolds without potential theory, Second edition, Springer-Verlag, 1979

[5] Friedrich T., Ivanov S., Parallel spinors and connections with skew-symmetric torsion in string theory. Asian J. Math., 2002, 6, 303-336

[6] Friedrich T., Ivanov S., Almost contact manifolds, connections with torsion, and parallel spinors. J. Reine Angew. Math., 2003, 559, 217-236

[7] Ganchev G., Borisov A., Note on the almost complex manifolds with Norden metric, C. R. Acad. Bulgare Sci., 1986, 39, 31-34

[8] Ganchev G., Gribachev K., Mihova V., B-connections and their conformal invariants on conformally Kaehler manifolds with B-metric, Publ. Inst. Math. (Beograd) (N.S.), 1987, 42(56), 107-121

[9] Ganchev G., Ivanov S., Characteristic curvatures on complex Riemannian manifolds, Riv. Mat. Univ. Parma, 1992, (5) 1, 155-162 
[10] Ganchev G., Mihova V., Canonical connection and the canonical conformal group on an almost complex manifold with B-metric, Annuaire Univ. Sofia Fac. Math. Inform., 1987, 81, 195-206

[11] Ganchev G., Mihova V., Gribachev K., Almost contact manifolds with B-metric, Math. Balkanica (N.S.), 1993, 7, 261-276

[12] Gates S. J., Hull C. M., Roček M., Twisted multiplets and new supersymmetric non-linear $\sigma$-models, Nucl. Phys. B, 1984, 248, 157-186

[13] Gauduchon P., Hermitian connections and Dirac operators, Boll. Unione Mat. Ital., 1997, 11, 257-288

[14] Gray A., Hervella L., The sixteen classes of almost Hermitian manifolds and their linear invariants, Ann. Mat. Pura Appl., 1980, 123, 35-58

[15] Gribacheva D., Natural connections on Riemannian product manifolds, C. R. Acad. Bulgare Sci., 2011, 64, 799-806

[16] Gribacheva D., Natural connections on conformal Riemannian P-manifolds, C. R. Acad. Bulgare Sci., 2012, 65, 581-590

[17] Gribacheva D., Mekerov D., Canonical connection on a class of Riemannian almost product manifolds, J. Geom., 2011, 102, 53-71

[18] Ivanov P., Ivanov S., $S U(3)$-instantons and $G_{2}, S p i n(7)$-heterotic string solitons, Comm. Math. Phys., 2005, 259, 79-102

[19] Ivanov S., Papadopoulos G., Vanishing theorems and string backgrounds, Classical Quant. Grav., 2001, 18, 1089-1110

[20] Lichnerowicz A., Un théorème sur les espaces homogènes complexes, Arch. Math., 1954, 5, 207-215

[21] Lichnerowicz A., Généralisation de la géométrie kählérienne globale, Coll. de Géom. Diff. Louvain, 1955, 99-122

[22] Manev M., Properties of curvature tensors on almost contact manifolds with B-metric, Proc. of Jubilee Sci. Session of Vasil Levski Higher Mil. School, Veliko Tarnovo, 1993, 27, 221-227

[23] Manev M., Contactly conformal transformations of general type of almost contact manifolds with B-metric. Applications, Math. Balkanica (N.S.), 1997, 11, 347-357

[24] Manev M., Natural connection with totally skew-symmetric torsion on almost contact manifolds with B-metric, Int. J. Geom. Methods Mod. Phys., 2012, 9, 1250044 (20 pages)

[25] Manev M., Gribachev K., Conformally invariant tensors on almost contact manifolds with B-metric, Serdica Math. J., 1994, 20, 133-147

[26] Manev M., Ivanova M., A natural connection on some classes of almost contact manifolds with B-metric, C. R. Acad. Bulgare Sci., 2012, 65, 429-436

[27] Manev M., Ivanova M., Canonical-type connection on almost contact manifolds with B-metric, Ann. Global Anal. Geom., 2013, 43, 397-408, DOI:10.1007/s10455-012-9351-z

[28] Manev M., Staikova M., On almost paracontact Riemannian manifolds of type $(n, n)$, J. Geom., 2001, 72, 108-114

[29] Mekerov D., A connection with skew-symmetric torsion and Kähler curvature tensor on quasi-Kähler manifolds with Norden metric, C. R. Acad. Bulgare Sci., 2008, 61, 1249-1256

[30] Mekerov D., Canonical connection on quasi-Kähler manifolds with Norden metric, J. Tech. Univ. Plovdiv Fundam. Sci. Appl. Ser. A Pure Appl. Math., 2009, 14, 73-86

[31] Nakova G., Zamkovoy S., Eleven classes of almost paracontact manifolds with semi-Riemannian metric of $(n+1, n)$, In: Adachi T., Hashimoto H., Hristov M. (Eds.), Recent Progress in Differential Geometry and its Related Fields, World Scientific Publ., Singapore, 2012, 119-136

[32] Naveira A.M., A classification of Riemannian almost product structures, Rend. Mat. Roma, 1983, $3,577-592$

[33] Staikova M., Gribachev K., Canonical connections and their conformal invariants on Riemannian P-manifolds, Serdica Math. J., 1992, 18, 150-161

[34] Strominger A., Superstrings with torsion, Nucl. Phys. B, 1986, 274, 253-284

[35] Tanaka N., On non-degenerate real hypersurfaces, graded Lie algebras and Cartan connections, Jpn. J. Math., 1976, 20, 131-190

[36] Tanno S., Variational problems on contact Riemannian manifolds, Trans. Amer. Math. Soc., 1989, 314, 349-379 
[37] Webster S. M., Pseudo-Hermitian structures on a real hypersurface, J. Differential Geom., 1978, 13, $25-41$

[38] Yano K., Differential geometry on complex and almost complex spaces, Pergamon Press, Oxford, 1965

[39] Yano K., Kon M., Structures on Manifolds, World Scientific, 1984

(M. Mane 1]) Department of Algebra and Geometry, Faculty of Mathematics and Informatics, Plovdiv University, 236 Bulgaria Blvd, Plovdiv 4027, Bulgaria

E-mail address: mmanev@uni-plovdiv.bg

(M. Ivanova) Department of Informatics and Mathematics, Faculty of Economics, Trakia University, Student Campus, Stara Zagora 6000, Bulgaria

E-mail address: mivanova@uni-sz.bg

${ }^{1}$ the corresponding author 